\title{
Les apports des Plans cancer à la cancérologie
}

\section{What Cancer Plans have brought to oncology}

\author{
Pr A. Buzyn \\ (C) Springer-Verlag France et INCa 2014
}

Résumé Portés au plus haut niveau de l'État, par les présidents de la République, les Plans cancer français prônent une approche intégrée de la lutte contre la maladie, couvrant à la fois le champ de la recherche, de la prévention, de l'organisation des soins et de l'après-cancer. Première stratégie nationale et globale de lutte contre les cancers, le Plan cancer 2003-2007 a permis de structurer le paysage de la cancérologie française et de garantir une qualité et une sécurité minimale des soins apportés aux malades à travers le territoire. Il a également organisé la coordination des acteurs, à l'échelon régional et interrégional, dans le domaine des soins et de la recherche en particulier. Le Plan cancer 2009-2013 a mis l'accent sur la personnalisation des prises en charge et sur le déploiement des innovations thérapeutiques, notamment en matière de médecine de précision. Ce deuxième Plan a également ouvert le chantier de la lutte contre les inégalités face à la maladie, qui sera au cœur de la mise en œuvre du Plan cancer 2014-2019, avec trois objectifs majeurs : la diminution de l'incidence des cancers, la baisse de la mortalité et l'amélioration de la qualité de vie des personnes touchées.

Mots clés Plan cancer - Lutte contre le cancer - Cancérologie · Prévention · Dépistage · Organisation des soins · Prise en charge personnalisée $\cdot$ Médecine de précision.

\begin{abstract}
French Cancer Plans, supported at the highest level of the State by successive Presidents of the Republic, promote an integrated approach of the fight against the disease that covers the areas of research, prevention, care and survivorship. As a first comprehensive cancer strategy, the Cancer Plan 2003-2007 both structured the field of oncology and ensured quality and safety of care for patients across the country. It also led to coordinate actors at regional and interregional levels, specifically in care and research areas. The Cancer Plan 2009-2013 focused on personalized care and deployed therapeutic innovations, particularly for precision
\end{abstract}

\section{Pr A. Buzyn $(\bowtie)$}

Présidente de l'Institut national du cancer

Institut national du cancer, 52 avenue André Morizet

F-92513 Boulogne-Billancourt Cedex medicine. The latter also launched the fight against cancer health inequalities, which is the priority of the Cancer Plan 2014-2019, pursued through three main objectives: reduce cancer incidence, lower mortality, and improve the quality of life of patients and survivors.

Keywords French Cancer Plan - Fight against cancer • Oncology · Prevention · Screening · Care · Personalized care · Precision medicine.

Faisant suite aux États généraux du cancer organisés par la Ligue nationale contre le cancer en 1998, la France a décidé de se doter d'une politique coordonnée de lutte contre cette maladie, fléau de santé majeur de notre société. Cette politique de lutte contre les cancers affiche depuis son origine deux particularités qui la distinguent des autres plans de santé publique et des approches internationales. D'une part, les Plans cancer français portent depuis le début une approche intégrée de la lutte contre la maladie, couvrant à la fois le champ de la prévention, celui de l'organisation des soins et l'appui à la recherche. Cette transversalité de l'approche a permis des synergies entre actions et la mise en œuvre d'initiatives innovantes propres à notre pays. D'autre part, les Plans cancer ont toujours été portés au plus haut niveau de l'État par les présidents de la République qui se sont succédé, leur donnant une dimension sociétale et médiatique particulière, un financement conséquent, et donc un statut à part parmi les plans de santé publique dont se dotent habituellement les États. Cette volonté politique particulièrement affirmée de faire de la lutte contre le cancer une priorité nationale a eu comme corollaire la responsabilisation des acteurs et l'obligation de résultat. Un Plan cancer présidentiel est jugé à l'aune des financements engagés et des ambitions projetées.

\section{Le Plan cancer 2003-2007 : première stratégie globale de lutte contre le cancer}

Ainsi, le président Jacques Chirac lançait en 2003 le premier Plan cancer, d'une durée de cinq ans, qui avait pour but de structurer le paysage de la cancérologie française et de garantir une sécurité minimale des soins apportés aux malades. Parmi 
les actions structurantes, ce Plan a notamment permis à la France de se doter d'un organisme dédié à la coordination des actions de lutte contre le cancer, l'Institut national du cancer (INCa), créé par la loi de santé de 2004. L'INCa a comme particularité d'être un groupement d'intérêt public (GIP). Ce statut, peu fréquent pour une agence sanitaire ou scientifique de l'État, lui permet de réunir au sein de son conseil d'administration (CA) l'ensemble des acteurs investis dans la lutte contre les cancers : les administrations des ministères en charge de la Santé et en charge de la Recherche (DGS, DGOS, DGRI), les quatre principales fédérations hospitalières (Fédération Unicancer, FHF, FHP, FEHAP), les trois principales caisses d'assurance maladie (CNAMTS, MSA, RSI), les deux principaux EPST de recherche en biologie et santé (Inserm et CNRS), les deux principales associations caritatives œuvrant dans ce champ (Fondation ARC pour la recherche sur le cancer et Ligue nationale contre le cancer, agréée pour représenter les usagers du système de santé) et des personnalités qualifiées (professionnels de santé, et un représentant des agences régionales de santé). Cette représentativité de l'ensemble des parties prenantes au sein du CA de l'INCa légitime son action et son rôle de coordination auprès des acteurs.

Le premier Plan a également permis d'organiser une coordination des acteurs à l'échelon territorial en créant les réseaux régionaux de cancérologie (RRC), les centres de coordination en cancérologie (3C), et les sept cancéropôles. Ces derniers ont eu notamment pour rôle d'animer et d'accompagner la dynamique régionale et interrégionale pour coordonner les efforts de recherche, ce qui a permis une réelle progression de la visibilité de la recherche française sur le cancer.

Dans les mesures notables visant à l'amélioration de la sécurité des soins, a été introduite l'obligation, pour les établissements prenant en charge des malades atteints de cancer, de répondre à des critères de qualité spécifiques à cette prise en charge. Parmi eux, figure l'obligation de discuter tout nouveau cas de cancer en réunion de concertation pluridisciplinaire $(\mathrm{RCP})$. Cette action est non seulement très emblématique et sécurisante pour les malades, leur garantissant d'être traités selon les standards du moment, mais cette confrontation des points de vue entre spécialistes participe également à la formation et à l'amélioration des pratiques professionnelles. La mise en place du dispositif d'annonce est une autre mesure emblématique d'amélioration de la qualité de la prise en charge, qui reconnaît là le traumatisme que représente l'annonce d'un diagnostic de cancer pour la personne concernée. Ce dispositif vise à garantir l'information, l'écoute et l'accompagnement dans le respect de la personne.

Parmi les critères d'autorisation établis par le premier Plan cancer, figurait également la notion de seuil minimum d'activité, garantissant aux patients d'être pris en charge par des équipes ayant une compétence spécifique dans le traitement des cancers. La mise en œuvre de ces critères d'autorisation, établis par l'INCa, et dont les ARS sont en charge de vérifier l'application sur le terrain, a entraîné une réduction du nombre d'établissements prenant en charge les personnes atteintes de cancer qui est passé de 2200 à 900 en quelques années.

Enfin, ce Plan s'est engagé de façon volontariste en faveur d'une meilleure prévention, notamment en proposant des mesures fortes de lutte contre le tabagisme en prévention primaire et en actant, dès 2004, la mise en place du dépistage organisé du cancer du sein en prévention secondaire.

Par le chemin tracé, ce premier Plan a permis aux acteurs de prendre pleinement conscience de la nécessité de s'organiser, de répondre à certaines contraintes et de devoir rendre compte des pratiques au bénéfice des patients.

\section{Le Plan cancer 2009-2013 : personnaliser les prises en charge}

Le deuxième Plan cancer, présenté par le président Nicolas Sarkozy en 2009, était structuré selon cinq axes (recherche, prévention, dépistage, soins et après-cancer). Tenant compte des acquis du précédent Plan en termes d'organisations structurelles, il a permis de compléter et conforter le dispositif par des organisations fonctionnelles répondant à des besoins spécifiques. La notion de personnalisation des prises en charge a été introduite et est devenue incontournable dans la pratique de la cancérologie. Ainsi, chaque patient se devait d'être informé de son parcours de soins par la remise d'un programme personnalisé de soins et de l'après-cancer (PPS et PPAC). Des organisations spécifiques ont ainsi été confortées pour l'oncopédiatrie, l'oncogériatrie, et pour les cancers rares avec 23 réseaux identifiés spécifiques à une pathologie. La nécessité de prendre en compte la qualité de vie des patients a été reconnue, garantissant notamment l'accès aux soins de support. Mais tout n'a pas abouti. Ce Plan affirmait la nécessité de lutter contre les inégalités face aux cancers, qui se sont creusées ces dernières années dans notre pays, et ce vaste chantier ne saurait être résolu en l'espace d'un Plan. De même, le Plan préconisait de renforcer le rôle central du médecin généraliste dans le parcours des personnes avant, pendant et après la maladie mais n'y associait pas de mesures précises et cet objectif n'a pas été atteint.

À l'inverse, l'accès aux innovations a été l'un des acquis majeurs de ce deuxième Plan. Ainsi, la France est maintenant à l'avant-garde de la médecine de précision. La prise en compte des caractéristiques génétiques des tumeurs, permettant l'accès aux traitements ciblés, s'est largement déployée grâce à la mise en place des 28 plateformes de génétique moléculaire accessibles à tous les malades sur le territoire ; le Plan a notablement renforcé les outils dédiés à la recherche clinique par la mise en place des équipes mobiles de recherche clinique (EMRC), par la labellisation de 16 centres d'essais cliniques de phase précoce $\left(\mathrm{CLIP}^{2}\right)$ et d'intergroupes coopérateurs. L'ensemble de ces mesures 
a généré une progression de la recherche clinique française en cancérologie, qu'elle soit à promotion académique ou industrielle, à contre-courant de ce qui a été observé pour la plupart des disciplines au cours de cette même période.

Cette description des mesures les plus emblématiques n'est bien entendu pas exhaustive des réalisations et des avancées que ces Plans ont générées. Mais nombre de difficultés persistent pour les personnes malades et les aidants, et cette maladie reste la première cause de décès dans notre pays, et notamment de décès prématurés et évitables, justifiant le lancement d'un troisième Plan cancer par le président François Hollande le 4 février dernier.

\section{Le Plan cancer 2014-2019 : des objectifs de santé publique ambitieux}

La question de l'opportunité d'un nouveau Plan cancer a été soulevée par certains. Mais l'analyse des acquis des Plans précédents démontre qu'outre l'apport de chacune des mesures prises individuellement, qui participent à l'amélioration de notre système de santé parfois même au-delà du champ du cancer, la construction d'un nouveau Plan induit une dynamique particulière. Les Plans français successifs sont regardés comme des modèles du genre en Europe en raison des modifications des prises en charges qu'ils ont permises et des innovations qu'ils ont favorisées. Peut-être expliquent-ils en partie les bons résultats en termes de survie des cancers observés en France par rapport à d'autres pays. Un Institut dédié à la coordination des actions face aux cancers, et qui est donc en responsabilité de les faire aboutir, est possiblement aussi l'une des clefs de leur mise en œuvre. Mais au-delà de ces avancées, le travail fourni pour l'élaboration d'un Plan par les parties prenantes (professionnels de santé et de la recherche, associations de malades ou d'usagers, administrations), la confrontation des points de vue, la définition d'objectifs ou de méthodes qui font consensus, aboutissent, in fine, à un contrat moral avec les usagers et les malades auquel adhère l'ensemble des acteurs. Un Plan est donc un engagement collectif vis-à-vis de la société, qui nous mobilise et qui nous oblige. Ce dernier Plan cancer 2014-2019, copiloté par le ministre en charge de la Santé et le ministre en charge de la Recherche, est ainsi structuré autour de trois objectifs principaux de santé que sont la diminution de l'incidence des cancers, la baisse de la mortalité et l'amélioration de la qualité de vie des personnes touchées, et d'un objectif d'efficience des organisations. Il affiche des cibles ambitieuses dans le champ de la prévention, de l'innovation et de la recherche, de la qualité des soins, et de l'accompagnement dans l'après-cancer. La mobilisation de tous les acteurs autour de ces objectifs à atteindre devrait permettre de franchir encore un pas en faveur de l'excellence des pratiques et des parcours, et garantir à notre pays de rester un modèle dans la lutte contre cette maladie.

\section{Le Plan cancer 2014-2019}

Le Plan a été construit selon une structure par grands objectifs de santé, déclinant à la fois des actions de santé et de recherche, autour d'une approche transversale de la lutte contre les inégalités et des pertes de chance face aux cancers.

L'ambition première du Plan est de guérir plus de personnes malades, en favorisant des diagnostics précoces et en garantissant l'accès de tous à une médecine de qualité et aux innovations.

Au-delà de l'amélioration des soins et des pratiques médicales, le Plan cancer propose une prise en charge globale de la personne, tenant compte de l'ensemble de ses besoins pour préserver la continuité et la qualité de vie pendant et après la maladie.

Les cancers sont aussi la première cause de mortalité évitable. Au regard des connaissances actuelles, on estime que près d'un décès par cancer sur deux pourrait être évité. Ainsi, le Plan investit dans la prévention pour réduire significativement le nombre de nouveaux cas de cancers. Enfin, il soutient la recherche fondamentale, source des progrès à venir.

À ces ambitions s'ajoute la volonté d'optimiser le pilotage et les organisations de la lutte contre les cancers, pour une meilleure efficience, en y associant pleinement les personnes malades et les usagers du système de santé.

À travers chacune des actions qu'il met en ouvre, le Plan cancer s'attaque résolument aux inégalités face à la maladie : en portant une attention particulière aux personnes les plus vulnérables, en luttant contre les pertes de chance lors de la prise en charge, en garantissant un accès égalitaire à l'innovation et aux essais cliniques, et en évitant que les conséquences économiques et sociales de la maladie n'aggravent les difficultés des personnes malades.

Le Plan, décliné en 17 objectifs opérationnels, s'inscrit dans la mise en ouvre de la Stratégie nationale de santé et de l'Agenda stratégique pour la recherche, le transfert et l'innovation « France-Europe 2020 ».

Le Plan cancer 2014-2019 est téléchargeable sur le site de l'INCa www.e-cancer.fr 